\title{
Mutations of TCF12, encoding a basic-helix-loop-helix partner of TWIST1, are a frequent cause of coronal craniosynostosis
}

\author{
Vikram P Sharma ${ }^{1,2,20}$, Aimée L Fenwick ${ }^{1,20}$, Mia S Brockop ${ }^{3,4,20}$, Simon J McGowan ${ }^{5}$, \\ Jacqueline A C Goos ${ }^{6,7}$, A Jeannette M Hoogeboom ${ }^{8}$, Angela F Brady ${ }^{9}, \mathbf{N}$ u Owase \\ Jeelani $^{10}$, Sally Ann Lynch ${ }^{11}$, John B Mulliken ${ }^{12}$, Dylan J Murray ${ }^{13}$, Julie M Phipps ${ }^{1}$, \\ Elizabeth Sweeney ${ }^{14}$, Susan E Tomkins ${ }^{15}$, Louise C Wilson ${ }^{16}$, Sophia Bennett ${ }^{17}$, Richard J \\ Cornall $^{17}$, John Broxholme ${ }^{18}$, Alexander Kanapin ${ }^{18}$, WGS500 ${ }^{19}$, David Johnson ${ }^{2}$, Steven A \\ Wall $^{2}$, Peter J van der Spek ${ }^{7}$, Irene M J Mathijssen ${ }^{6}$, Robert E Maxson ${ }^{3}$, Stephen R F \\ Twigg $^{1,20}$, and Andrew O M Wilkie ${ }^{1,2}$
}

${ }^{1}$ Clinical Genetics Group, Weatherall Institute of Molecular Medicine, University of Oxford, Oxford, UK. ${ }^{2}$ Craniofacial Unit, Oxford University Hospitals NHS Trust, Oxford, UK. ${ }^{3}$ USC/Norris Cancer Center, Los Angeles, USA. ${ }^{4}$ Université Paris-Sud, Orsay, France. ${ }^{5}$ Computational Biology Research Group, Weatherall Institute of Molecular Medicine, University of Oxford, Oxford, UK. ${ }^{6}$ Department of Plastic and Reconstructive Surgery, Erasmus MC, University Medical Center Rotterdam, The Netherlands. ${ }^{7}$ Department of Bioinformatics, Erasmus MC, University Medical Center Rotterdam, The Netherlands. ${ }^{8}$ Department of Clinical Genetics, Erasmus MC, University Medical Center Rotterdam, The Netherlands. ${ }^{9}$ Kennedy Galton Centre, North West London Hospitals NHS Trust, London, UK. ${ }^{10}$ Department of Neurosurgery, Great Ormond Street Hospital for Children NHS Trust, London, UK. ${ }^{11}$ National Centre for Medical Genetics, Our Lady's Children's Hospital, Dublin, Ireland. ${ }^{12}$ Children's Hospital, Boston, USA. ${ }^{13}$ National Paediatric Craniofacial Centre, Children's University Hospital, Dublin, Ireland. ${ }^{14}$ Department of Clinical Genetics, Liverpool Women's NHS Foundation Trust, Liverpool, UK. ${ }^{15} \mathrm{Clinical}$ Genetics Department, Musgrove Park Hospital, Taunton, UK. ${ }^{16}$ North East Thames Regional Genetics Service, Great Ormond Street Hospital for Children NHS Trust, London, UK. ${ }^{17}$ MRC Human Immunology Unit, Weatherall Institute of Molecular Medicine, University of Oxford, Oxford, UK. ${ }^{18}$ Wellcome Trust Centre for Human Genetics, University of Oxford, Oxford, UK.

\section{Abstract}

Craniosynostosis, the premature fusion of the cranial sutures, is a heterogeneous disorder with a prevalence of $\sim 1$ in 2,200 (refs. 1,2). A specific genetic etiology can be identified in $\sim 21 \%$ of cases $^{3}$, including mutations of TWIST1, which encodes a class II basic helix-loop-helix (bHLH) transcription factor, and causes Saethre-Chotzen syndrome, typically associated with coronal

Correspondence should be addressed to R.E.M. (Robert.Maxson@med.usc.edu) or A.O.M.W. (andrew.wilkie@imm.ox.ac.uk).

${ }_{19} \mathrm{~A}$ list of WGS500 names and affiliations is provided in the Supplementary Note.

${ }^{20}$ These authors contributed equally to this work.

AUTHOR CONTRIBUTIONS S.R.F.T., R.E.M. and A.O.M.W. conceived the project. V.P.S., A.L.F., M.S.B. and S.R.F.T. performed experimental analyses. S.B. and R.J.C. performed immune function tests. S.J.McG, J.B., A.K., P.D. and WGS500 co-ordinated or performed bioinformatics analyses. V.P.S., J.A.C.G., A.J.M.H., A.F.B., O.J., S.A.L., J.B.M., D.J.M., J.M.P., E.S., S.E.T., L.C.W., D.J., S.A.W., P.J.S., I.M.J.M. and A.O.M.W. recruited patient samples and collected clinical information. V.P.S., A.L.F., R.E.M., S.R.F.T. and A.O.M.W. drafted the manuscript.

URLs. ANNOVAR, http://www.openbioinformatics.org/annovar; GBrowse2, http://gmod.org/wiki/GBrowse; MRC-Holland, www.mrc-holland.com/pages/indexpag.html; PolyPhen-2, http://genetics.bwh.harvard.edu/pph2; SAMtools, http:// samtools.sourceforge.net.

Accession codes. All cDNA numbering of TCF12 follows NCBI reference NM_207037.1, starting with A of the ATG initiation codon $(=1)$. We used NM_207040.1 to design primers to the alternatively spliced first exon (9A). The genomic reference sequence is available from NC_000015.9. 
synostosis $^{4-6}$. Starting with an exome sequencing screen, we identified 38 heterozygous TCF12 mutations in 347 samples from unrelated individuals with craniosynostosis. The mutations predominantly occurred in patients with coronal synostosis and accounted for $32 \%$ and $10 \%$ of subjects with bilateral and unilateral pathology, respectively. TCF12 encodes one of three class I E-proteins that heterodimerize with class II bHLH proteins such as TWIST1. We show that TCF12 and TWIST1 act synergistically in a transactivation assay, and that mice doubly heterozygous for loss-of-function mutations in Tcf 12 and Twist 1 exhibit severe coronal synostosis. Hence, the dosage of TCF12/TWIST1 heterodimers is critical for coronal suture development.

Our exome sequencing approach focused on bilateral coronal craniosynostosis (Fig. 1a) because of previous evidence that this pathological group is loaded with cases of monogenic etiology ${ }^{3}$. We examined the variant lists from whole exome data ${ }^{7}$ of seven unrelated patients with bilateral coronal synostosis, negative for previously described mutations ${ }^{8}$, for genes showing non-synonymous changes in two or more samples. Two samples had different heterozygous frameshift mutations in TCF12 (transcription factor 12; also known as HEB, HTF4 and ALF1) ${ }^{9-11}$; one (Family \#19) had a single nucleotide deletion and the other (\#30) a 4-nucleotide deletion (details in Supplementary Table 1). The mutations were confirmed by dideoxy-sequencing of the original DNA samples (primary sequence results for all families are shown in Supplementary Fig. 1). In addition a text search of the exome sequence data revealed a novel T>A variant in TCF12 in a third sample (\#22), located 20 nucleotides upstream of the start of exon 17. This variant was predicted to generate a cryptic splice acceptor site that was confirmed experimentally (Supplementary Fig. 2a). Thus, we had identified different heterozygous disruptive mutations of TCF12 in 3 of 7 samples from patients with bilateral coronal synostosis. We interrogated other exome or whole genome sequencing projects on craniosynostosis that we were undertaking concurrently: this revealed further TCF12 mutations in two siblings with bilateral coronal synostosis and their clinically unaffected mother (Family \#20, which we had erroneously analyzed assuming recessive inheritance), and in two unrelated individuals $(\# 11,16)$ with clinical diagnoses of Saethre-Chotzen syndrome who did not have identified mutations in the TWIST1 gene ${ }^{4-6}$.

To confirm these findings and explore the clinical consequences, we carried out dideoxysequencing of TCF12 in patients with different types of craniosynostosis in whom mutations had not previously been identified. In the initial panel of 287 unrelated samples, we identified 18 with pathogenic mutations; all mutation-positive individuals had coronal synostosis. To replicate our findings, we sequenced an independent Dutch cohort of 50 samples with coronal synostosis and identified 14 further mutations. Overall we observed an extreme bias for TCF12 mutations to occur in association with coronal synostosis (Table 1): 22 of $69(32 \%)$ bilateral coronal and 14 of $141(10 \%)$ unilateral coronal cases had mutations, but none of 93 cases with isolated metopic, sagittal or lambdoid synostosis $\left(P=1 \times 10^{-6}\right.$, Fisher's exact test). The remaining two TCF12 mutation-positive cases had combinations of sagittal and either uni- or bilateral coronal synostosis, so coronal synostosis was present in all 38 index patients. In our extended Oxford birth cohort ${ }^{3}$ with minimum 5-year follow-up (cases born in calender years 1998-2006), we found TCF12 mutations in 4/402 (1.0\%; $95 \%$ confidence interval $0.3-2.5 \%$ ) patients. Hence, mutations in TCF12 are quantitatively important in craniosynostosis overall (Supplementary Fig. 3).

The mutations identified in TCF12 comprize 14 nonsense, 15 frameshift, 7 splicing and 2 missense changes, strongly suggesting a loss-of-function mechanism (Fig. 2). This is consistent with previous reports ${ }^{12}$ of patients with cytogenetically identified chromosome 15 deletions (predicted to include TCF12 at 15q21.3) in whom craniosynostosis was present, although no whole exon deletions of TCF12 were apparent in our patient panel using a 
multiplex ligation-dependent probe amplification (MLPA) assay. In cases with frameshift or splice site mutations, analyses of mRNA extracted from various cell types demonstrated relative under-expression of the mutant compared to the wild-type allele, consistent with nonsense-mediated decay leading to haploinsufficiency (Supplementary Fig. 2 and Supplementary Table 2). The mutations showed a clear enrichment in the $3^{\prime}$ half of TCF12, with all but one located between exons 10 and 19. However the remaining mutation (c. $526+1 \mathrm{G}>\mathrm{A}$ ), which caused skipping of exon 7 (Supplementary Fig. 2d), is specific to the longer TCF12 transcript, as the alternative transcription start site ${ }^{13}$ lies downstream of this position (exon 9A, Fig. 2a). The two missense mutations (p.Leu624Pro and p.Gln638Glu) are located within highly conserved residues of the bHLH DNA-binding/dimerization domain (Supplementary Fig. 4a and b); mutations of other bHLH proteins often cluster within this region, examples including the class I protein TCF4 (Pitt-Hopkins syndrome) ${ }^{14}$ and class II protein TWIST1 (ref. 6). Using a transactivation reporter assay ${ }^{15}$, we found that the combination of native TCF12 and TWIST1 proteins exhibited a synergistic effect on activation compared with either protein individually, but this was reduced by $65-76 \%$ in the presence of the TCF12 missense mutations p.Leu624Pro or p.Gln638Glu (Supplementary Fig. 4c).

Of 36 families for which additional samples were available, the mutation was shown to have arisen de novo in 14 (Supplementary Fig. 1 and Supplementary Table 1), providing further evidence that the TCF12 mutations are causative of the coronal synostosis phenotype. In 23 of the families cascade testing identified 34 further mutation-positive individuals, only 16 of whom had craniosynostosis or other suspicious clinical features, indicating substantial (47\%) non-penetrance (Supplementary Table 1, and see Supplementary Fig. 5 for representative clinical photographs). There was no evidence to implicate somatic mosaicism in any of the non-penetrant cases, but preliminary analysis of the haplotype background around $T C F 12$ (which resides in a region of strong linkage disequilibrium) provides several indications that this contributes to variability in the phenotype (data not shown). Of 14 mutation-positive relatives diagnosed with craniosynostosis involving specific sutures, 12 had coronal synostosis and two had sagittal synostosis.

The clinical features associated with $T C F 12$-related craniosynostosis are detailed in Supplementary Table 3; representative subjects with bilateral coronal or unilateral coronal synostosis are illustrated in Fig. 1a and b, respectively. In 15 probands (39\%) a genetic cause was suspected because of a positive family history. In another 7 probands (18\%), clinical features (including dysmorphic appearance of face and external ears, and minor limb anomalies) were suggestive of Saethre-Chotzen syndrome, which had been excluded by negative TWIST1 mutation testing. The remaining patients $(16 / 38=42 \%)$ presented sporadically with apparently non-syndromic synostosis (Supplementary Table 1). No genotype-phenotype correlation was apparent. For patients recruited at Oxford, we compared the surgical trajectory ${ }^{3}$ of $T C F 12$-positive patients with other clinical and genetic categories. The TCF12 patients had a more benign course compared with those with either p.P250R-FGFR3 or TWIST1 mutations (Supplementary Fig. 6) and no TCF12-positive individuals were documented to have an interval onset of raised intracranial pressure following their initial surgical procedure(s). Most individuals had normal developmental attainment, but $10(14 \%)$ had significant developmental delay or learning disability, in two cases associated with autism (clinical features are summarized in Supplementary Table 4). Previous genetic data derived from homozygous mutant mice identified key roles for the orthologous $T c f 12$ gene in the development of the immune system ${ }^{13,16}$, but we elicited no history of infection susceptibility amongst TCF12 heterozygotes and there was no detectable reduction in monocyte, $\mathrm{NK}$, T or B cell subsets or immunoglobulin in 11 mutation-positive individuals. 
Supporting our transactivation data (Supplementary Fig. 4) it was previously reported that TCF12 forms heterodimers with TWIST1 (ref. 17), and that TWIST1 haploinsufficiency causes Saethre-Chotzen syndrome ${ }^{4-6}$. Our observation that haploinsufficiency of TCF12 in humans leads specifically to coronal synostosis suggests that the total quantity of TCF12/ TWIST1 heterodimers is a critical factor in coronal suture development. To test this hypothesis, we compared the phenotypes of the previously constructed individual mouse heterozygous null mutants (EIIa-Cre; Tcf $12^{\text {flox/+ }}$ and Twist $\left.1^{+/-}\right)^{16,18,19}$ with the compound heterozygotes (EIIa-Cre; Tcf1 $2^{\text {flox } /+} ;$ Twist $\left.^{+/-}\right)$. At 3 weeks of age, the EIIa-Cre; Tcf $12^{\text {flox/+ }}$ animals $(n=14)$ had normal coronal sutures, indicating that the coronal suture is less sensitive to Tcf12 dosage in mice than in humans (Fig. 1c and d); Twist $1^{+/-}$mice $(n=4)$ exhibited variable coronal synostosis, as previously reported (Fig. 1e) $)^{5,20}$. However the EIIa-Cre; Tcf $12^{\text {flox } /+} ;$ Twist $1^{+/}$compound heterozygotes $(n=6)$, which were born at near the expected Mendelian ratio (25\%), consistently exhibited severe coronal synostosis (Fig. 1f).

The discovery that haploinsufficiency of TCF12 causes coronal synostosis in humans, and that severe bilateral coronal synostosis occurs in mice with a 50\% dose of both the Tcf 12 and Twist 1 genes, highlights the key role of TCF12 acting with TWIST1, probably as a heterodimeric complex, for normal development of the coronal sutures. This heterodimer is likely to regulate specification of the boundary between neural crest and cephalic mesoderm ${ }^{20,21}$, and/or inhibit osteogenic differentiation via actions on RUNX2 (ref. 22), bone morphogenetic protein ${ }^{23}$ or fibroblast growth factor receptor ${ }^{17}$ signalling.

\section{Online Methods}

\section{Samples}

The clinical studies were approved by Oxfordshire Research Ethics Committee B (reference C02.143), Riverside Research Ethics Committee (reference 09/H0706/20), and the Medical Ethical Committee of the Erasmus University Medical Centre Rotterdam (MEC-2012-140). Written informed consent to obtain samples for genetics research was obtained from each child's parent or guardian. The clinical diagnosis of craniosynostosis was confirmed by computed tomographic scanning, and evidence of associated syndromic features sought by clinical examination. Venous blood was obtained for DNA and RNA extraction (collected into PAXgene Blood RNA tubes [Qiagen] for the latter procedure). Fibroblast cultures were established from skin biopsies obtained from the scalp incision at the time of surgical intervention. When clinically indicated, samples were tested for mutation hotspots in FGFR2, FGFR3 and TWIST1 (ref. 8), and significant chromosome aneuploidy was investigated using array comparative genomic hybridization; samples with identified mutations known to cause craniosynostosis were excluded. Seven samples from unrelated individuals with bilateral coronal synostosis were chosen for exome sequencing. These comprized five patients who were clinically non-syndromic and with negative family history; one patient who was clinically non-syndromic but reported to have two children with coronal synostosis; and one patient who was only the only individual in the family affected with craniosynostosis, but who had short thumbs that were also present in several additional family members from the two previous generations.

\section{Exome sequencing}

We used an Agilent SureSelect Human All Exon Kit (v.2; $44 \mathrm{Mb}$ ) to capture exonic DNA from a library prepared from $3 \mu \mathrm{g}$ of each proband's genomic DNA (extracted from whole blood). The enriched DNA was sequenced on an Illumina GAIIx platform with $51 \mathrm{bp}$ paired-end reads. We generated between $3.98 \mathrm{~Gb}$ and $4.56 \mathrm{~Gb}$ of sequence for each sample that, after mapping with Novoalign (Novocraft Technologies) to the hg19 genome and removal of artefacts using custom perl scripts, resulted in an average coverage of exons of 
between 33- and 44- fold. Variants were called using SAMtools and annotated using ANNOVAR and PolyPhen-2. We removed all variants annotated in dbSNP132, and manually examined the gene lists for overlaps of two or more variants. Sequence data were visualized using GBrowse2.

\section{Analysis of TCF12}

Following the identification of candidate $T C F 12$ mutations in the exome sequence, we confirmed and extended this analysis by direct sequencing of genomic PCR amplification products, MLPA, and analysis of cDNA products. Oligonucleotides and experimental conditions are provided in Supplementary Table 5. Our initial screening panel mostly comprized samples recruited in Oxford ${ }^{3}$ and other craniofacial units within the UK, and the replication panel comprized samples obtained in Rotterdam, the Netherlands. PCR amplification was performed in a volume of $20 \mu \mathrm{l}$, containing $15 \mathrm{mM}$ TrisHCl (pH 8.0), 50 $\mathrm{mM} \mathrm{KCl}, 2.5 \mathrm{mM} \mathrm{MgCl} 2,100 \mu \mathrm{M}$ each dNTP, $0.4 \mu \mathrm{M}$ primers, and 0.5 units of Amplitaq Gold polymerase (Applied Biosystems), with or without $10 \%$ dimethysulfoxide as indicated. Cycling conditions consisted of an $8 \mathrm{~min}$ denaturation step at $94^{\circ} \mathrm{C}$, followed by 35 cycles of $94^{\circ} \mathrm{C}$ for $30 \mathrm{~s}$, annealing at $65^{\circ} \mathrm{C}$ (unless otherwise indicated in Supplementary Table 5) for $30 \mathrm{~s}$ and extension at $72^{\circ} \mathrm{C}$ for $30 \mathrm{~s}$, with a final extension at $72^{\circ} \mathrm{C}$ for $10 \mathrm{~min}$. The amplification products were sequenced using the BigDye Terminator v3.1 cycle sequencer system (Applied Biosystems). All mutations were re-confirmed either by restriction digest of $5 \mu \mathrm{l}$ of PCR product or by repeat dideoxy-sequencing of an independent PCR product. In addition to the mutations featured in Supplementary Table 1, we identified two previously unreported variants in single individuals that are currently of uncertain pathogenic significance, c.630T $>$ G (p.S210R) and c.982A $>$ G (p.N328D).

Copy number variation was analyzed by MLPA performed using synthetic oligonucleotide probes designed to TCF12 according to protocols available from MRC-Holland. Fragments were analyzed by capillary electrophoresis using an ABI 3130 containing POP-7 polymer. Peaks were visualized using Gene Mapper v3.7 (Applied Biosystems). Normal MLPA results were obtained in 226 craniosynostosis samples negative for intragenic TCF12 mutations. For studies of splicing and mRNA stability, RNA was extracted from whole blood and fibroblasts using Trizol reagent. cDNA was synthesized using the Fermentas RevertAid First Strand Synthesis kit with random hexamer primers according to the manufacturer's instructions. The microsatellites used to confirm sample relationships were D3S1311, D4S403, D5S2027, D6S1610, D7S519, D9S158, D10S548, D11S898, D13S1265, D14S280, D16S415 and D18S474.

\section{Immune function tests}

Peripheral blood mononuclear cells (PBMCs) were isolated from whole blood by density gradient centrifugation in cell separation tubes (Sigma, Gillingham, UK), washed three times in sterile PBS and rested overnight in RPMI 1640 media, supplemented with $10 \%$ fetal calf serum, $1 \% \mathrm{~L}$-glutamine, $1 \%$ penicillin-streptomycin solution, $50 \mu \mathrm{M} \beta$-mercaptoethanol (all from Sigma) and 1\% HEPES buffer (GibcoBRL). Monocytes, NK cells and naïve, effector and memory $\mathrm{T}$ and $\mathrm{B}$ cell subsets were enumerated by flow cytometry of PBMCs stained with anti-human antibodies against CD3, CD4, CD19, CD14, CCR7, CD45RO, CD3, CD62L, CD16, CD56, CD25, IgD, IgM, CD10 and CD27 purchased from BD Biosciences (San Jose, California). Total IgM, IgA and IgG antibody titres were measured by ELISA.

\section{Transactivation analysis of missense mutations}

We obtained a full length TCF12 cDNA clone (pCMV6-XL5, encoding HEB $\beta$ ) from OriGene and synthesized the TWIST1 clone (pCMV-Twist) in-house. The pCaSpeR- 
Tinman::lacZ (pTinE1/E2/E3) construct (containing three Tinman E-boxes comprizing two $5^{\prime}$-CATGTG-3' and one $5^{\prime}$-CATATG- $3^{\prime}$ ) was used as a bHLH protein-responsive reporter and placZ (containing no E-boxes) as a control (both constructs were gifts from ErnstMartin Füchtbauer, Aarhus University, Denmark $)^{15}$. PCR mutagenesis was used to introduce the c.1871T $>\mathrm{C}$ and c. $1912 \mathrm{C}>\mathrm{G}$ mutations into the TCF12 cDNA using primers indicated in Supplementary Table 5; mutated cDNAs were fully sequence verified.

The HT1080 human cell line was cultured in high-glucose Dulbecco's modified Eagle's medium (Invitrogen) supplemented with 10\% fetal calf serum. Cells were transfected in triplicate with $1 \mu \mathrm{g}$ of reporter plasmid, $1 \mu \mathrm{g}$ each of TWIST1 and TCF12 constructs or the corresponding empty expression vectors, using Promega FuGENE 6 transfection reagent according to manufacturer's protocols. Transfections were carried out when cells were $\sim 50 \%$ confluent in 6-well plates. The cells were harvested after $48 \mathrm{~h}$, and $\beta$-galactosidase assays were performed in triplicate on $5 \mu \mathrm{l}$ of cellular extract using the Galacto-Star system (Applied Biosystems). The missense mutations were modelled in Protein Workshop onto the structure of murine E47/NeuroD1 (PDB accession 2QL2) ${ }^{26}$.

\section{Analysis of mouse mutants}

The mouse mutants analyzed were $T c f 12^{\text {flox }}$ (obtained on a mixed C57BL/6/129 background as a gift of Dr Yuan Zhuang, Duke University) ${ }^{16}$, EIIa-Cre (purchased from The Jackson Laboratory (B6.FVB-Tg(EIIa-cre)C5379Lmgd/J ) ${ }^{18}$ and Twist1 (C57BL/6 background) ${ }^{19}$. The EIIa-Cre transgene was detected using primers Cre-F and Cre-R (Supplementary Table 5); Tcf1 $2^{\text {flox }}$ and Twist 1 were genotyped as described ${ }^{16,19}$. Heads of 21-day-old postnatal (P21) mice were skinned and stained for mineralized bone with Alizarin Red S (80 mg/l in $1 \% \mathrm{KOH}$ ) for 3 days. The skulls were then cleared and stored in 100\% glycerol. P21 mouse skulls were scanned using a $\mu \mathrm{CT}$ system (SCANCO microCT 50, Scanco Medical AG, Switzerland) and analyzed using Amira software (Visage Imaging, Germany). All scans were conducted at an energy setting of $70 \mathrm{kVp}$, current intensity of $200 \mu \mathrm{A}$, integration time of $500 \mathrm{~ms} /$ projection, 0.24 degree rotational step (DRS) and a field of view (FOV) of 15.1 $\mathrm{mm}$. The scans were performed at a $10 \mu \mathrm{m}$ isotropic resolution.

\section{Supplementary Material}

Refer to Web version on PubMed Central for supplementary material.

\section{Acknowledgments}

We thank all the families for their participation, Sue Butler for cell culture, John Frankland and Tim Rostron for DNA sequencing, Samantha Knight for coordinating array CGH, Lorna Gregory and the High-Throughput Genomics core at WTCHG for exome sequencing, Russell Evans for review of anesthetic records, Warwick Baggley for clinical photography, Ans van den Ouweland for genetic testing, Ernst-Martin Füchtbauer for constructs and Yuan Zhuang for the gift of the Tcf $12^{\text {flox }}$ mutant. This work was funded by the NIHR Biomedical Research Centre Oxford (R.J.C.), Oxford University Clinical Academic Graduate School and Oxfordshire Health Services Research Committee (V.P.S.), Oxford Craniofacial Unit Charitable Fund (V.P.S.), Thames Valley Comprehensive Local Research Network (J.M.P.), The Dutch Center for Translational Molecular Medicine (P.J.S.), Carolien Bijl Foundation (J.A.C.G.), NIH (R01DE016320 and R01DE019650 to R.E.M.) and the Wellcome Trust (093329 to S.R.F.T. and A.O.M.W.).

\section{References}

1. Lajeunie E, Le Merrer M, Bonaïti-Pellie C, Marchac D, Renier D. Genetic study of nonsyndromic coronal craniosynostosis. Am. J. Med. Genet. 1995; 55:500-504. [PubMed: 7762595]

2. Boulet SL, Rasmussen SA, Honein MA. A population-based study of craniosynostosis in metropolitan Atlanta, 1989-2003. Am. J. Med. Genet. 2008; 146A:984-991. [PubMed: 18344207] 
3. Wilkie AOM, et al. Prevalence and complications of single-gene and chromosomal disorders in craniosynostosis. Pediatrics. 2010; 126:e391-400. [PubMed: 20643727]

4. Howard TD, et al. Mutations in TWIST, a basic helix-loop-helix transcription factor, in SaethreChotzen syndrome. Nat. Genet. 1997; 15:36-41. [PubMed: 8988166]

5. El Ghouzzi V, et al. Mutations of the TWIST gene in the Saethre-Chotzen syndrome. Nat. Genet. 1997; 15:42-46. [PubMed: 8988167]

6. Jabs, EW. TWIST and the Saethre-Chotzen syndrome. In: Epstein, CJ.; Erickson, RP.; WynshawBoris, A., editors. Inborn Errors of Development. The Molecular Basis of Clinical Disorders of Morphogenesis. 2nd ed. Oxford University Press; Oxford, England: 2008. p. 474-481.

7. $\mathrm{Ng} \mathrm{SB}$, et al. Exome sequencing identifies the cause of a mendelian disorder. Nat. Genet. 2010; 42:30-35. [PubMed: 19915526]

8. Johnson D, Wilkie AOM. Craniosynostosis. Eur. J. Hum. Genet. 2011; 19:369-376. [PubMed: 21248745]

9. Hu JS, Olson EN, Kingston RE. HEB, a helix-loop-helix protein related to E2A and ITF2, that can modulate the DNA-binding ability of myogenic regulatory factors. Mol. Cell. Biol. 1992; 12:10311042. [PubMed: 1312219]

10. Nielsen AL, Pallisgaard N, Pedersen FS, Jørgensen P. Murine helix-loop-helix transcriptional activator proteins binding to the E-box motif of the Akv murine leukemia-virus enhancer identified by cDNA cloning. Mol. Cell. Biol. 1992; 12:3449-459. [PubMed: 1321336]

11. Gan T-I, et al. Genomic organization of human TCF12 gene and spliced mRNA variants producing isoforms of transcription factor HTF4. Cytogenet. Genome Res. 2002; 98:245-248. [PubMed: 12826747]

12. Hiraki Y, et al. Craniosynostosis in a patient with a de novo $15 \mathrm{q} 15-\mathrm{q} 22$ deletion. Am. J. Med. Genet. 2008; 146A:1462-1465. [PubMed: 18449934]

13. Wang D, et al. The basic helix-loop-helix transcription factor HEBAlt is expressed in pro-T cells and enhances the generation of T cell precursors. J. Immunol. 2006; 177:109-119. [PubMed: 16785505]

14. Whalen S, et al. Novel comprehensive diagnostic strategy in Pitt-Hopkins syndrome: Clinical score and further delineation of the TCF4 mutational spectrum. Hum. Mutat. 2012; 33:64-72. [PubMed: 22045651]

15. Laursen KB, Mielke E, Iannaccone P, Fuchtbauer EM. Mechanism of transcriptional activation by the proto-oncogene Twist1. J. Biol. Chem. 2007; 282:34623-34633. [PubMed: 17893140]

16. Wojciechowski J, Lai A, Kondo M, Zhuang Y. E2A and HEB are required to block thymocyte proliferation prior to pre-TCR expression. J. Immunol. 2007; 178:5717-5726. [PubMed: 17442955]

17. Connerney J, et al. Twist1 dimer selection regulates cranial suture patterning and fusion. Dev. Dyn. 2006; 235:1345-1357. [PubMed: 16502419]

18. Lakso M, et al. Efficient in vivo manipulation of mouse genomic sequences at the zygote stage. Proc. Natl. Acad. Sci. USA. 1996; 93:5860-5865. [PubMed: 8650183]

19. Chen ZF, Behringer RR. twist is required in head mesenchyme for cranial neural tube morphogenesis. Genes Dev. 1995; 9:686-699. [PubMed: 7729687]

20. Merrill AE, et al. Cell mixing at a neural crest-mesoderm boundary and deficient ephrin-Eph signaling in the pathogenesis of craniosynostosis. Hum. Mol. Genet. 2006; 15:1319-1328. [PubMed: 16540516]

21. Chai Y, Maxson RE Jr. Recent advances in craniofacial morphogenesis. Dev. Dyn. 2006; 235:2353-2375. [PubMed: 16680722]

22. Bialek P, et al. A Twist code determines the onset of osteoblast differentiation. Dev Cell. 2004; 6:423-435. [PubMed: 15030764]

23. Hayashi M, et al. Comparative roles of Twist-1 and Id1 in transcriptional regulation by BMP signaling. J. Cell. Sci. 2007; 120:1350-1357. [PubMed: 17374642]

24. Aronheim A, Shiran R, Rosen A, Walker MD. The E2A gene-product contains two separable and functionally distinct transcription activation domains. Proc. Natl. Acad. Sci. USA. 1993; 90:80638067. [PubMed: 8367464] 
25. Markus M, Du ZM, Benezra R. Enhancer-specific modulation of E protein activity. J. Biol. Chem. 2002; 277:6469-6477. [PubMed: 11724804]

26. Longo A, Guanga GP, Rose RB. Crystal structure of E47-NeuroD1/Beta2 bHLH domain-DNA complex: heterodimer selectivity and DNA recognition. Biochemistry. 2008; 47:218-229.

[PubMed: 18069799] 

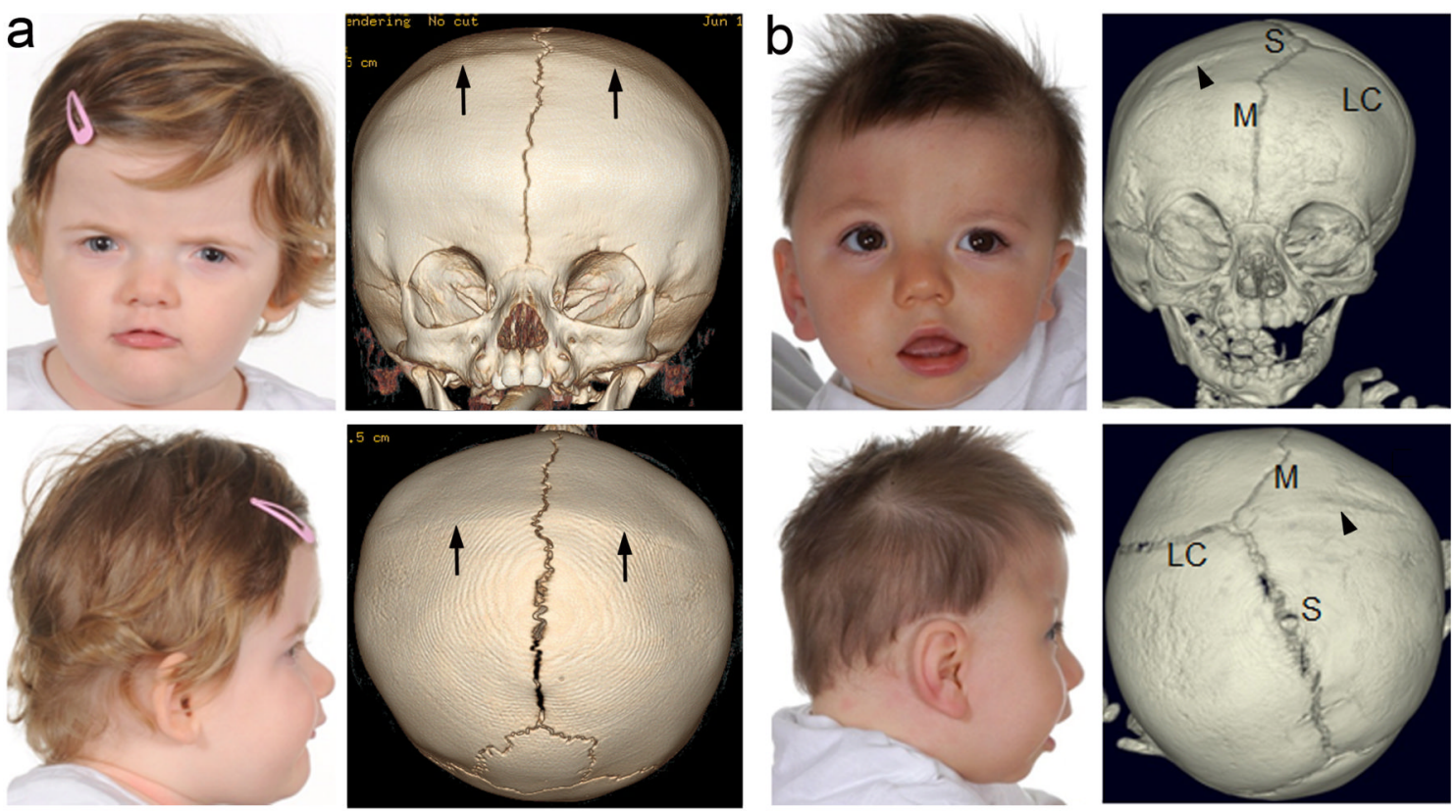

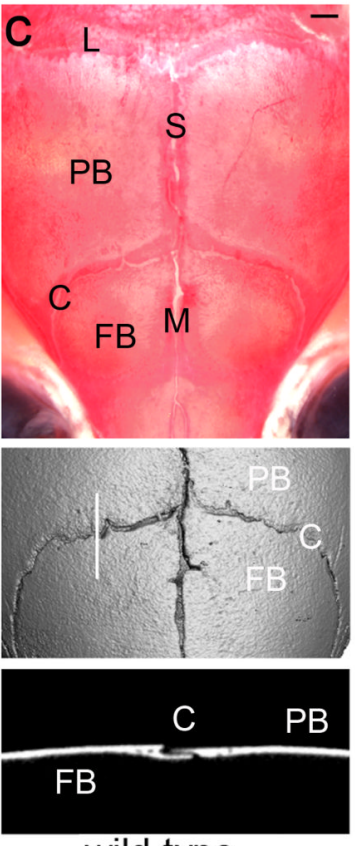

wild-type
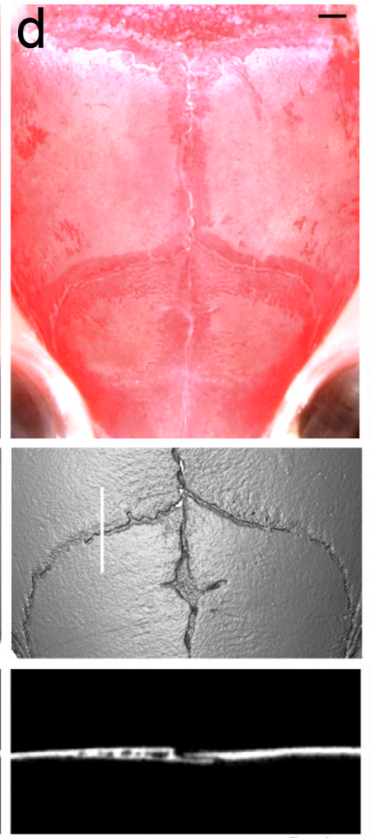

Ella-Cre;Tcf12 flox/+

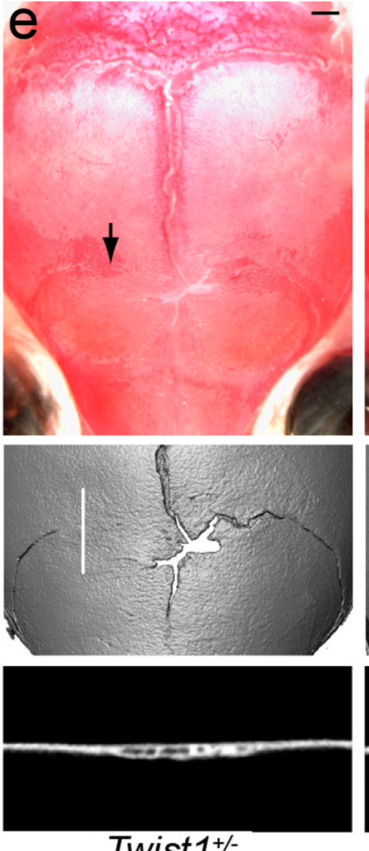

Twist 1/-
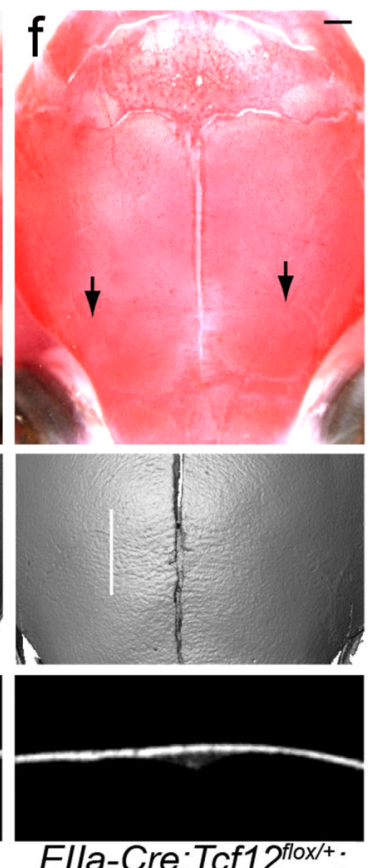

Twist ${ }^{\text {+1- }}$

Figure 1.

Phenotype associated with TCF12/Tcf12 haploinsufficiency in humans and mice. (a,b) Clinical presentation of individuals with $T C F 12$ mutations, showing facial appearance (left) and computed tomography (CT) images of head (right). Written permission was obtained to publish the clinical photographs. (a) Bilateral coronal synostosis in proband \#30: note high forehead and brachycephaly at the age of $13 \mathrm{mo}$ and, on CT images at the age of $4 \mathrm{mo}$, bilateral fusion of the coronal sutures associated with bony ridging (arrows). (b) Right unilateral coronal synostosis in proband \#35: note elevation of right orbit and shifting of midface and mandible to left at the age of 10 mo. CT images at the age of 4 mo demonstrate complete fusion of the coronal suture on the right (arrowhead), an open coronal suture on 
the left (LC) and relative angulation of the metopic (M) and sagittal (S) sutures. (c-f) Severe bilateral coronal synostosis in EIIa-Cre; Tcf1 $2^{\text {flox } /+} ; T_{\text {wist }} 1^{+/}$combination mutants. Panels show representative images of postnatal day (P)21 skulls stained with Alizarin Red S (top), 3-dimensional $\mu \mathrm{CT}$ reconstructions (middle) and 2-dimensional $\mu \mathrm{CT}$ images of sagittal slices $(10 \mu \mathrm{m})$ through the coronal suture (bottom; planes of section are indicated by white lines in 3-dimensional images). Note normal coronal sutures in wild-type (c) and EIIaCre; $T c f 12^{f l o x /+}$ skull (d), partial unilateral coronal synostosis in $T$ wist $1^{+/-}$skull (e, arrow) and complete bilateral coronal synostosis in EIIa-Cre; Tcf $12^{\text {flox } /+} ;$ Twist $^{+/-}$skull (f, arrows). $\mathrm{C}$, coronal suture; $\mathrm{FB}$, frontal bone; $\mathrm{M}$, metopic suture; L: lambdoid suture; $\mathrm{PB}$, parietal bone; S, sagittal suture. Scale bars: $1 \mathrm{~mm}$. 
a

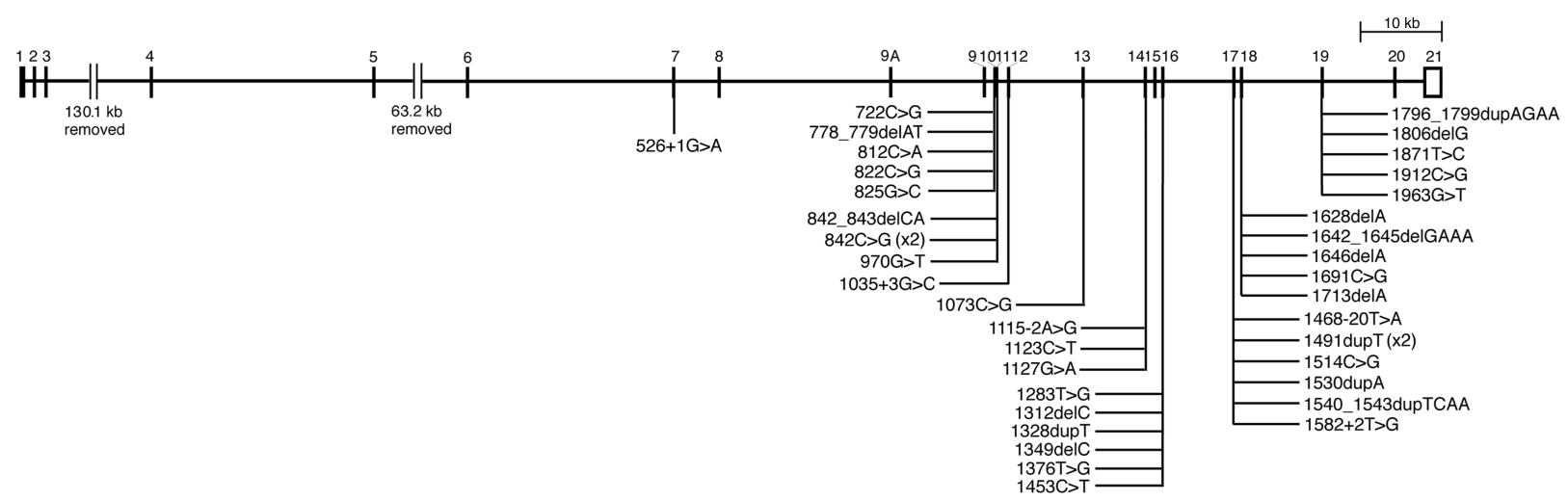

b

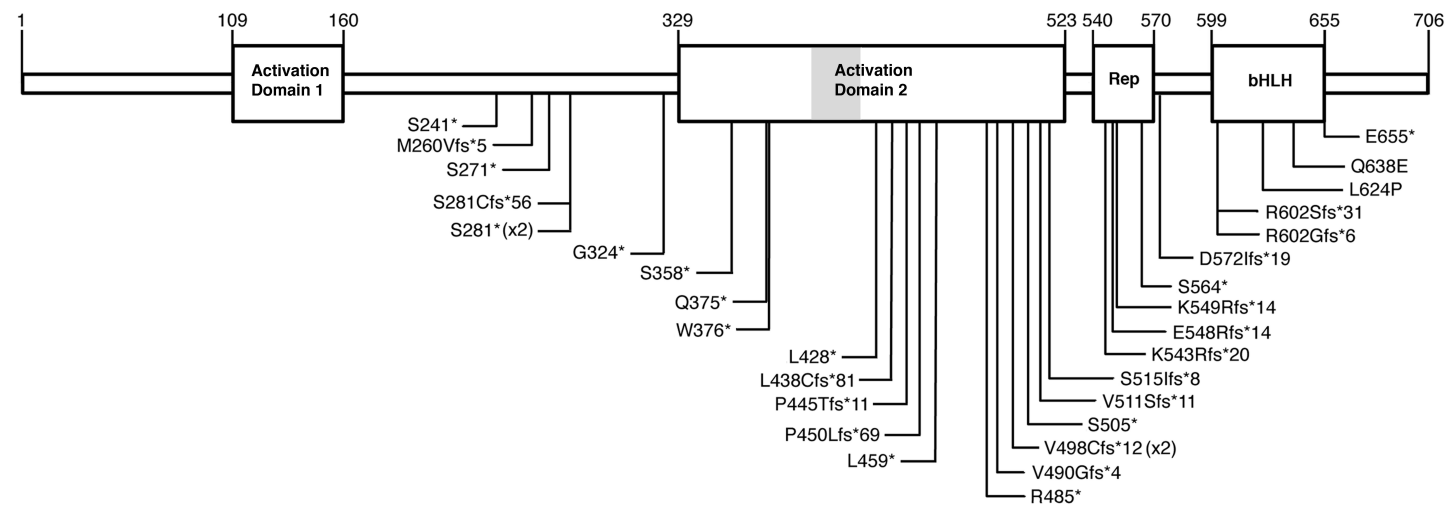

Figure 2.

Structure of TCF12 and encoded protein, showing location of mutations identified in coronal synostosis. (a) Gene structure. The major transcript comprizes 21 exons; inclusion or exclusion of exon 15 by alternative splicing generates proteins of 706 or 682 amino acids, termed $\mathrm{HEB} \beta$ and $\mathrm{HEBa}$, respectively. Alternative transcription starting at exon $9 \mathrm{~A}$ generates a different protein (HEBAlt) of 512 amino acids ${ }^{13}$. (b) Domains identified in the HEB $\beta$ protein (portion encoded by exon 15 shaded gray). The highly conserved bHLH region lies near the $\mathrm{C}$-terminus. Functional studies previously defined Activation Domains 1 and 2 (ref. 24) and the Rep domain ${ }^{25}$. Identical mutations identified in independent families are denoted (x2); note that mutations predicted to affect splicing are omitted from the protein cartoon. 
Table 1

Identification of TCF12 mutations in different categories of craniosynostosis

\begin{tabular}{|c|c|c|c|c|c|c|}
\hline & $\begin{array}{c}\text { Non- } \\
\text { syndromic }\end{array}$ & & Syndromic & & Combined & \\
\hline & Total & $\begin{array}{c}T C F 12 \\
\text { mutation } \\
\text { positive }\end{array}$ & Total & $\begin{array}{c}T C F 12 \\
\text { mutation } \\
\text { positive }\end{array}$ & Total & $\begin{array}{c}T C F 12 \\
\text { mutation } \\
\text { positive }\end{array}$ \\
\hline Metopic & 32 & 0 & 14 & 0 & 46 & 0 \\
\hline Sagittal & 32 & 0 & 7 & 0 & 39 & 0 \\
\hline $\begin{array}{l}\text { Unilateral } \\
\text { coronal }\end{array}$ & 115 & 9 & 26 & 5 & 141 & 14 \\
\hline $\begin{array}{l}\text { Bilateral } \\
\text { coronal }\end{array}$ & 28 & 5 & 41 & 17 & 69 & 22 \\
\hline $\begin{array}{l}\text { Uni- or } \\
\text { bilateral } \\
\text { lambdoid }\end{array}$ & 8 & 0 & 0 & 0 & 8 & 0 \\
\hline Multisuture & 19 & 1 & 19 & 1 & 38 & 2 \\
\hline $\begin{array}{l}\text { Sutures } \\
\text { not } \\
\text { specified }\end{array}$ & 2 & 0 & 4 & 0 & 6 & 0 \\
\hline Combined & 236 & 15 & 111 & 23 & 347 & 38 \\
\hline
\end{tabular}

